\title{
Internetbasierte Psychotherapie und E-Mental-Health
}

\author{
Franz Caspar $^{\mathrm{a}}$ Thomas Berger ${ }^{\mathrm{a}}$ Winfried Lotz-Rambaldi ${ }^{\mathrm{b}} \quad$ Fritz Hohagen $^{\mathrm{b}}$ \\ ${ }^{a}$ Klinische Psychologie und Psychotherapie, Universität Bern, Schweiz \\ ${ }^{\mathrm{b}}$ Zentrum für Integrative Psychiatrie, Campus Lübeck, Universität zu Lübeck, Deutschland
}

«Internettherapie» ist nicht nur unter Laien, sondern auch unter Therapeuten mit Vorurteilen behaftet: «Damit können doch viele Patienten nichts anfangen!», «Super Sache, vor allem für Patienten in entlegenen Gebieten!», «...und was, wenn ein Patient suizidal wird?», «Mir würde das nonverbale Verhalten der Patienten fehlen!», «Das kann ja nur bei jüngeren Patienten funktionieren!», «Wie soll denn da eine vernünftige therapeutische Beziehung entstehen?», usw.

Bedenkt man, dass Aktualität unter anderem dadurch charakterisiert ist, dass Meinungen auseinander gehen, dann ist Internettherapie eindeutig ein aktuelles Thema. Nur die wenigsten Therapeuten dürften in Zukunft davon gänzlich unberührt bleiben. Die meisten Patienten werden eines nicht allzu fernen Tages vor der Entscheidung für eine bestimmte Art von Therapie auch über internetbasierte Alternativen informiert und über mögliche Vor- und Nachteile aufgeklärt werden. Schon heute kann man jederzeit von Patienten gefragt werden: «Was halten Sie eigentlich von Internettherapie?». Fest steht: Die Umgebung hat sich gewandelt. Immer mehr Menschen natürlich auch jenen mit psychischen Problemen - ist der Zugang zum Internet vertraut, das ihnen zusätzliche Möglichkeiten zur Bewältigung ihres Alltags und zur Lösung ihrer Probleme bietet. Qualifizierte psychosoziale Hilfs- und Beratungsangebote im Internet nehmen zu, lassen sich aber nicht immer leicht von denen unseriöser Anbieter unterscheiden.

Kazdin und Blase [2011] weisen weiter darauf hin, dass wir als professionelle Gemeinschaft gar keine Chance haben, allein mit traditioneller Psychotherapie den gesamten Interventionsbedarf bei psychischen Problemen zu decken. Internettherapie ist einer der alternativen Ansätze mit hohem Potenzial, bestehende Versorgungslücken zu verkleinern, wie in den einzelnen Beiträgen dieses Heftes dargelegt wird.

Uns schien das Thema Internettherapie jedenfalls wichtig genug, um dazu ein Schwerpunktheft der VerhaltenstheraPIE zu gestalten. Schnell wurde klar, dass «Internet-Psychotherapie» im engeren Sinne einige relevante Anschlussthemen wie Nachsorge, Online-Supervision oder SMS-Interventionen außen vor lassen würde. So haben wir das Thema $\mathrm{zu}$ «Internettherapie und E-Mental-Health» erweitert; wir gehen hier jedoch zunächst auf Ersteres ein.

Die Leser sind gefordert, vorschnelle Urteile etwas zurückzuhalten, und seien sie intuitiv noch so verständlich. So liegt für einen erfahrenen Praktiker nichts näher als sich zu fragen, ob Internettherapie mit den eigenen Patienten funktionieren würde; sowohl bezüglich der Akzeptanz als auch der Prozesse und Ergebnisse. Aber: Die Patienten, die auf Internettherapie ansprechen, sind nicht unbedingt dieselben, die den Weg in eine Face-to-Face (F2F)-Therapie, also in ein normales Psychotherapie-Setting finden. Ein weiterer Anteil von «Internet-Patienten», dessen Höhe uns erstaunte, hat bereits enttäuschende Erfahrungen unterschiedlicher Art mit F2F-Therapie gemacht. Welche Wege führen in die Internettherapie unter Berücksichtigung der Zugangswege zu F2F-Therapie? Wie könnten Übergangsindikationen aussehen, wenn die erstgewählte Therapieform nicht hilft oder sich zu viele unerwünschte Nebenwirkungen zeigen?

Patientenpräferenzen hängen stark von der aktuellen Bekanntheit der Angebote ab, können sich aber auch schnell ändern, wenn im Laufe einer Therapie die Erfahrung gemacht wird, dass diese hilft [Berger, 2013] - aber dazu muss man sich natürlich erst einmal auf eine Therapie eingelassen haben. Dass Internettherapie alle «abholt», die sich nicht auf eine Therapie mit einem leibhaftigen Therapeuten einlassen, wäre zu optimistisch: Es gibt sicher eine größere Gruppe von Menschen mit psychischen Problemen, denen Internettherapie helfen könnte, die sich aber auf keinerlei Form von Therapie einlassen. Auch Internettherapie ist kein Zaubermittel, was den Zugang zu Patienten betrifft.

Ein ganz spannendes Thema ist die therapeutische Beziehung. Belege für deren Wichtigkeit in F2F-Therapien stammen überwiegend aus korrelativen Studien. Korrelationen beruhen aber auf Variation, d.h. ohne schlechte Therapie-

\section{KARGER \\ Fax +497614520714}

Information@Karger.com

www.karger.com (c) 2013 S. Karger GmbH, Freiburg

1016-6262/13/0233-0137\$38.00/0

Accessible online at:

www.karger.com/ver
Prof. Dr. Franz Caspar

Abteilung Klinische Psychologie und Psychotherapie

Universität Bern

Gesellschaftsstrasse 49, 3012 Bern, Schweiz

franz.caspar@psy.unibe.ch 
beziehungen (und ohne schlechte Ergebnisse) kann es keine größeren Korrelationen geben. Das scheint oft vergessen zu werden, wenn Therapeuten an konkrete F2F-Therapien denken, in denen die Beziehung eine entscheidende Rolle spielte. Es ist zwar nur schwer vorstellbar, dass ganz spezifische, starke Beziehungserlebnisse sich so auch in Internettherapien abspielen könnten. Aber dass sich aus Sicht der Patienten auch in Internettherapien mit Therapeutenkontakt erstaunlich intensive Beziehungen entwickeln können, ist inzwischen gut belegt und entspricht auch den Alltagserfahrungen vieler Internetnutzer, die Chat- oder E-Mail-basierte Beziehungen pflegen. Vielleicht ist es sogar leichter, zu einem entsprechend den eigenen Bedürfnissen imaginierten Therapeuten eine gute Beziehung zu entwickeln als zu einem realen. Zudem: Wo es keine oder nur eine minimale reale Beziehung gibt, gibt es keinen narzisstischen oder anderen Missbrauch, keine schlechte Beziehung, die Prozess und Ergebnis belasten könnten. Wenn man dennoch annimmt, auf der Beziehungsebene würde etwas fehlen, dann würden die äquivalenten Outcome-Ergebnisse von Internet- und F2F-Therapien darauf hinweisen, dass sich andere Faktoren zugunsten der Ersteren auswirken. Ein solcher Faktor könnte sein, dass ein ambivalenter Patient weiß, dass es auf ihn und seinen Einsatz ankommt. Er wird insgeheim nicht darauf hoffen können, dass der Therapeut ihn auffängt, wodurch er viele Chancen verpassen würde, sich selbst zu helfen. Wir wissen schließlich aus der regulären Therapieforschung, dass der Einsatz des Patienten eine ganz zentrale Variable ist.

Es gibt einschlägige Angebote, zu denen es kaum Alternativen gibt, z.B. Internettherapie für Traumatisierte während des Irakkrieges [Wagner und Knaevelsrud 2011]. Gerade im Trauma-Bereich mahnen die negativen Ergebnisse für Debriefing-Prozeduren allerdings, dass es nicht nur darum geht zu entscheiden, welcher von 2 möglichen Therapieansätzen besser ist. Auch konkurrenzlose Therapien haben zu belegen, dass positive Prä-Post-Veränderungen stattfinden, mindestens aber, dass sie nicht schaden.

Die einzelnen Beiträge in diesem Heft sind an der Empirie orientiert. Dabei öffnet sich zwischen Einzelfallstudien mit N = 2 und zusammenfassenden Darstellungen einer Vielzahl von Originalstudien ein breites Spektrum von Methoden, die dem Forschungsstand zu einzelnen Fragen angepasst sind.

Boettcher et al. [2013] fassen eindrucksvolle Belege für die Wirksamkeit von Internettherapie bei Angststörungen zusammen. Was wirksam ist, führt allerdings nicht nur zu einer Verschiebung des Mittelwertes, sondern auch zu einer Varianzerweiterung. Die Autoren stellen die Forderung nach differenzierterer Analyse, unter anderem zu Prädiktoren und Wirkmechanismen. Dazu sind große Patientenzahlen nötig, die bei F2F-Therapien oft nicht zu realisieren sind. Ein spezifischer Vorteil von Internettherapien ist, dass Forscher bei diesen leichter innerhalb einer überschaubaren Zeit zu einer größeren Stichprobe kommen. Dürfen wir hoffen, dass den berechtigten Forderungen schneller nachgekommen werden kann als in F2F-Therapien?
Klein und Berger [2013] beschäftigen sich dann mit einer zweiten diagnostischen Gruppe: Patienten mit Depressionen. Sie heben unter anderem hervor, dass der Einsatz von Internettherapien es realistischer macht, Patienten mit Psychotherapie zu versorgen - der von den meisten Patienten gegenüber Medikation präferierten und dem Stand der Empirie entsprechend in Leitlinien empfohlenen Behandlungsform. Weiter betonen sie die Bedeutung einer sorgfältigen diagnostischen Abklärung wie sie etwa von gut ausgebildeten Hausärzten geleistet werden kann, im Vergleich zu reiner Internettherapie mit Direktzugang.

Andersson und Hedman [2013] gehen der Frage nach, ob die guten Effekte, die für «guided internet-based cognitive behavior therapy» gefunden wurden, auch in regulären klinischen Settings fortbestehen. Sie fassen dazu Ergebnisse aus verschiedenen Studien zusammen und diskutieren eine ganze Reihe von Aspekten, die in der Routineversorgung beachtet werden müssen.

Klinitzke et al. [2013] beschäftigen sich mit internetbasierter Therapie nach Verlust eines Kindes in der Schwangerschaft. Es handelt sich um eine Schreibtherapie via Internet. Besonders interessant fanden wir die Anregung der Suche/ Aktivierung realer sozialer Unterstützung in der letzten Phase der Internettherapie - widerspricht dies doch der stereotypen Erwartung, Internettherapie fände nur in der engen Welt zwischen Patient und Computer statt.

In weiteren Beiträgen verlassen wir den Bereich der Therapie im engeren Sinne und kommen zu Aufrechterhaltung und Nachsorge: Brakemeier et al. [2013] beschreiben CBASP@home, ein internetbasiertes SituationsanalysenTraining zur Stabilisierung des Therapieerfolgs nach stationärer Therapie für chronisch depressive Patienten. Vorgestellt werden 2 Einzelfälle. Der Ansatz entspricht ganz der Haltung von CBASP-Begründer McCullough: Dieser vergleicht chronische Depression mit Diabetes, einer Krankheit, die lebenslang Aufmerksamkeit und Maßnahmen erfordert: Auch da erfolgt die Insulinbehandlung ja nicht durch den Arzt, sondern der Patient übernimmt die tägliche Behandlung selbst.

Ebenfalls der Nachsorge, in diesem Fall per SMS und bei Patientinnen mit Bulimia nervosa, ist der Beitrag von Bauer et al. [2013] gewidmet: Sie berichten weniger Rückfälle und weniger Inanspruchnahme neuer Therapien, wenn Patientinnen eine SMS-Nachsorge erhalten.

Jakob et al. [2013] erweitern und ergänzen die Thematik in eine andere Richtung: Live-Supervision: Vom Einwegspiegel zur videobasierten Online-Supervision. Seit Langem wird mit Einwegscheiben und direkter Intervention in den Therapieraum hinein (vgl. auch «reflecting team» [Andersen 1990]) Live-Supervision praktiziert. Beim «bug in the ear» (das Bild vom Ohrhörer kennen wir von Türstehern und Bodyguards) wurden auch technische Mittel der Tonübertragung eingesetzt. Alle diese Ansätze haben den Nachteil, intrusiv zu sein und damit Therapien unter Um- 
ständen eher zu verschlechtern als zu verbessern. Der Bugin-the-eye-Ansatz hat den Vorteil, dass der Therapeut die schriftliche Rückmeldung auf einem Monitor im Therapieraum beachten kann - sofern dies im Moment nicht gerade stören würde. Die Präsenz des Supervisors via Internet macht Live-Supervision aus Zeit- und Kostengründen deutlich realistischer. Die Akzeptanz scheint gut, aber weitere Untersuchungen sind nötig, die auch (Selbst-)Selektionseffekte berücksichtigen.
Und schließlich werden in einem Interview mit Gerhard Andersson [2013] Erfahrungen mit der Internettherapie in Schweden diskutiert, einem Land, in dem dieses neue Therapieformat intensiv erforscht und teilweise in die Regelversorgung integriert ist.

Insgesamt bietet dieses Heft einen guten Einblick in den Stand der Diskussion, an vielen Stellen zudem auch in die aktuellen Entwicklungen, und schließlich Blicke in die Zukunft, der wir uns alle optimistisch stellen sollten.

\section{Literatur}

Andersen T: Das reflektierende Team: Dialoge und Dialoge über Dialoge. Modernes Lernen, Dortmund, 1990.

Andersson G, Hedman E: Effectiveness of guided inter net-based cognitive behavior therapy in regular clinical settings. Verhaltenstherapie 2013;23:140-148.

Bauer S, Okon E, Meermann R, Kordy H: SMS-Nachsorge: Sektorenübergreifende Versorgung für Patientinnen mit Bulimia nervosa. Verhaltenstherapie 2013;23:204-210.

Berger T: Internet-based treatments - experiences from Sweden. An interview with Gerhard Andersson. Verhaltenstherapie 2013;23:211-211-214.

Boettcher J, Carlbring P, Renneberg B, Berger T: Internet-based interventions for social anxiety disorder. Verhaltenstherapie 2013;23:160-169.
Brakemeier E-L, Marchner J, Gutgsell S, Engel V, Radtke M, Tuschen-Caffier B, Normann C, Berking M: CBASP@home: Ein internetbasiertes Situationsanalysen-Training zur Stabilisierung des Therapieerfolgs nach stationärer Therapie für chronisch depressive Patienten. Verhaltenstherapie 2013;23:190-203.

Jakob M, Weck F, Bohus M: Live-Supervision: Vom Einwegspiegel zur videobasierten Online-Supervision. Verhaltenstherapie 2013;23:170-180.

Kazdin A, Blase SL: Rebooting psychotherapy research and practice to reduce the burden of mental illness. Perspectives on Psychological Science 2011; 6:21-37.
Klein P, Berger T: Internetbasierte psychologische Behandlung bei Depressionen. Verhaltenstherapie 2013;23:149-159.

Klinitzke G, Dölemeyer R, Steinig J, Wagner B, Kersting A: Internetbasierte Therapie nach Verlust eines Kindes in der Schwangerschaft - Einfluss sozialer Unterstützung auf die Verarbeitung des Verlusts. Verhaltenstherapie 2013;23:181-189.

Wagner B, Knaevelsrud C: Traumatherapie über das Internet - neue Behandlungsmöglichkeiten in Kriegsund Krisengebieten. Psychotherapie im Dialog 2011; 12:167-170. 